\title{
The erratic mitochondrial clock: variations of mutation rate, not population size, affect mtDNA diversity across birds and mammals Benoit Nabholz*, Sylvain Glémin and Nicolas Galtier
}

\author{
Address: Université Montpellier 2 CNRS UMR 5554 - Institut des Sciences de l'Evolution Place E. Bataillon - CC064, 34095 Montpellier, France \\ Email: Benoit Nabholz* - benoit.nabholz@univ-montp2.fr; Sylvain Glémin - glemin@univ-montp2.fr; Nicolas Galtier - galtier@univ-montp2.fr \\ * Corresponding author
}

Published: 10 March 2009

BMC Evolutionary Biology 2009, 9:54 doi:|0.1 |86//47|-2|48-9-54

This article is available from: http://www.biomedcentral.com/I47I-2/48/9/54

(C) 2009 Nabholz et al; licensee BioMed Central Ltd.

This is an Open Access article distributed under the terms of the Creative Commons Attribution License (http://creativecommons.org/licenses/by/2.0), which permits unrestricted use, distribution, and reproduction in any medium, provided the original work is properly cited.
Received: 21 May 2008
Accepted: 10 March 2009

\begin{abstract}
Background: During the last ten years, major advances have been made in characterizing and understanding the evolution of mitochondrial DNA, the most popular marker of molecular biodiversity. Several important results were recently reported using mammals as model organisms, including (i) the absence of relationship between mitochondrial DNA diversity and life-history or ecological variables, (ii) the absence of prominent adaptive selection, contrary to what was found in invertebrates, and (iii) the unexpectedly large variation in neutral substitution rate among lineages, revealing a possible link with species maximal longevity. We propose to challenge these results thanks to the bird/mammal comparison. Direct estimates of population size are available in birds, and this group presents striking life-history trait differences with mammals (higher massspecific metabolic rate and longevity). These properties make birds the ideal model to directly test for population size effects, and to discriminate between competing hypotheses about the causes of substitution rate variation.

Results: A phylogenetic analysis of cytochrome $b$ third-codon position confirms that the mitochondrial DNA mutation rate is quite variable in birds, passerines being the fastest evolving order. On average, mitochondrial DNA evolves slower in birds than in mammals of similar body size. This result is in agreement with the longevity hypothesis, and contradicts the hypothesis of a metabolic rate-dependent mutation rate. Birds show no footprint of adaptive selection on cytochrome $b$ evolutionary patterns, but no link between direct estimates of population size and cytochrome $b$ diversity. The mutation rate is the best predictor we have of within-species mitochondrial diversity in birds. It partly explains the differences in mitochondrial DNA diversity patterns observed between mammals and birds, previously interpreted as reflecting Hill-Robertson interferences with the $\mathrm{W}$ chromosome.
\end{abstract}

Conclusion: Mitochondrial DNA diversity patterns in birds are strongly influenced by the wide, unexpected variation of mutation rate across species. From a fundamental point of view, these results are strongly consistent with a relationship between species maximal longevity and mitochondrial mutation rate, in agreement with the mitochondrial theory of ageing. Form an applied point of view, this study reinforces and extends the message of caution previously expressed for mammals: mitochondrial data tell nothing about species population sizes, and strongly depart the molecular clock assumption. 


\section{Background}

Animal mitochondrial DNA (mtDNA) evolution contrasts with nuclear evolution. Mitochondrial and nuclear genomes differ in many ways, such as total length, ploidy level, mode of inheritance, recombination rate, presence of introns, percentage of non-coding DNA, effective population size, and repair mechanisms, e.g. [1,2]. Among these particularities, hypermutability is one of the most striking features of animal mitochondria: the mtDNA mutation rate is typically one order of magnitude higher than the nuclear one $[1,3,4]$. The question of the origin and evolution of such high mutation rates is still open and debated $[5,6]$; and see $[7,8]$ for exceptions.

This high mutation rate is one of the reasons why mtDNA is a very popular marker for biodiversity studies. For example, it has been massively used to investigate intraspecific to intra-ordinal evolutionary relationship, and disentangle rapid speciation events in phylogenetic studies (e.g. see $[9,10]$ for a comparison of nuclear and mitochondrial markers). In recent years, mtDNA has been used to identify species using a standardized portion of the cytochrome oxydase I gene (COI), according to the socalled DNA barcoding approach [11]. The high mtDNA mutation rate, however, is also the source of frequent homoplasy, i.e., phylogenetic incongruence between sites of the molecule because of multiple mutations at the same site. Homoplasy complicates the use of mtDNA in phylogenetic $[9,10,12]$ and even population genetic [13] studies.

We recently reported several results related to mtDNA mutation dynamics in mammals. First, we showed that mtDNA substitution rates are extremely variable between species: they differ by two orders of magnitude between slow-evolving and fast-evolving mammalian lineages [14], definitively rejecting the famous " $2 \%$ per site per million year" calibration, which should not be generally trusted. We proposed that mutation rate variations are possibly linked to species longevity through the action of natural selection: too high a mutation rate would be deleterious in long-lived mammals because it could result in premature aging due to the accumulation of somatic mtDNA mutations [14], see also [15-17]. Secondly, we found that within-species cytochrome $b$ (cytb) nucleotide diversity is correlated with the mitochondrial mutation rate, as expected, but not with any life-history or ecological variable potentially related to population size, including body size, geographic range and conservation status [18]. This surprising pattern was apparently not explained by selective effects [18] (see also [19]), contrary to results obtained at the Metazoa level [20], especially invertebrates [21]. We therefore proposed that the lack of correlation between mtDNA diversity and potential indicators of population size in mammals is due to strong demo- graphic stochasticity. At any rate, the mutation rate is the best predictor we have of mitochondrial genetic diversity across mammalian species. Overall, these results highlight the importance of mutation rate variations in shaping mtDNA biodiversity patterns in mammals, and suggest that mutational effects should be carefully taken into account when analysing such data.

In this study, we extend our analysis of the evolutionary dynamics of mtDNA through the bird/mammal comparison. Our objective is dual. First, we want to check whether the mammalian results are specific to this group, or have any degree of generality. Secondly, we want to make use of the genetical and physiological specificities of birds to test various evolutionary hypothesis raised by previous studies. Birds are the perfect candidate to challenge the results obtained in mammals: like mammals, they include charismatic, well studied species, in which a large amount of genetic and biological data are available. Being relatively large, warm blooded vertebrates, birds are comparable to mammals in terms of physiology, ecology, and lifehistory. Birds, furthermore, show a number of peculiarities potentially relevant to mitochondrial evolution and diversity.

The first reason why birds are appropriate for a comparative approach is the availability of direct population size estimates obtained through global population surveys, especially for North American species [22]. Birds therefore provide the opportunity to directly test the relationship between population size and mitochondrial diversity, whereas in mammals we had to rely on ecological and life history traits, plausibly, but only supposedly, correlated to population size. We will therefore check whether the lack of relationship between mtDNA diversity and species abundance we reported in mammals is confirmed in birds, or was due to inappropriate measure of the effective population size.

Birds, secondly, present a genetic peculiarity: female is the heterogametic sex. Because it is strictly maternally transmitted, the avian mitochondrial genome is thus in full genetic linkage with the female-specific $\mathrm{W}$ chromosome [23]. Berlin et al. [24] proposed that the mtDNA diversity could therefore be reduced by Hill-Robertson interference: selective effects applying to loci linked to the $\mathrm{W}$ will affect mtDNA through hitch-hiking. This clever hypothesis has provoked some comments and reactions [24-27]. Hickey [26] questioned one of the most important arguments of Berlin et al. [24], namely the lower synonymous diversity in bird than in mammal mtDNA, arguing that mutation rate could be a confounding effect. An accurate estimation of mtDNA substitution rate variations in birds appears necessary to correctly interpret the patterns of mtDNA diversity in the bird/mammal comparison. 
Birds, finally, have quite high metabolic rates -1.5 to 2.5 times higher than mammals of similar sizes [28] -probably because of the high energetic demand of the flying locomotion. Paradoxically, birds species are strikingly long-lived as compared to their mammalian counterparts; on average, birds live three times longer than mammals of similar sizes $[28,29]$. Birds can be characterized as longlived homeotherms, a specificity they share with bats [30]. These peculiarities are useful to understand further the impact of metabolic rate and life-span on mtDNA substitution rates.

Three main hypotheses have been proposed to explain mtDNA substitution rate variations in mammals: the generation time hypothesis [30-33], the metabolic rate hypothesis [34,35], and the longevity hypothesis [14-17], presented above. The classical approach to test these hypotheses in a comparative framework is to correlate substitution rate variations with the relevant life history traits $[14,17,33]$ : female sexual maturity, basal mass-specific metabolic rate (or body mass, taken as a proxy), and maximum longevity. However, these life-history traits are strongly correlated with each other, so that the respective contributions of the three variables are difficult to disentangle. The contrast between the avian and mammalian physiologies provides a unique opportunity to discriminate between two of these competing models, namely the longevity and the metabolic rate hypotheses. According to the longevity hypothesis, birds should present, on average, lower mtDNA neutral substitution rates than mammals, while the reverse pattern is expected under the metabolic rate hypothesis.

To test these predictions and challenge the results we obtained in mammals, we propose to accurately estimate the lineage-specific neutral substitution rate variation of mtDNA in birds using the phylogenetic framework developed by [14]. Using this dataset, we want to check the rough $2 \% /$ site/Myr calibration. This calibration, originally estimated with an RFLP analysis on mammals by Brown et al. [3], was generalized to birds by a study in geese [36]. This calibration rapidly became a standard in ornithology studies, probably because of the scarcity of the fossil record (see [37,38] and [39] for a review). Recently, the reliability of this calibration was debated in various studies either supporting [40-42] or rejecting it $[39,43]$. We will also ask which of metabolic rate and longevity is the best predictor of mutation rate variations in warm-blooded vertebrates. Finally, we will estimate the mitochondrial genetic diversity (synonymous and nonsynonymous) on a wide taxonomic range in birds to test for the neutrality assumption and investigate the link between population size, mutation rate, genetic hitch-hiking, and mtDNA diversity.

\section{Results \\ Substitution rate variation and its determinants}

To infer the substitution rate variation of a large number of bird species, we need to estimate divergence dates, and the numbers of nucleotide substitutions which occurred during these divergences (branch lengths). The divergence dates are obtained thanks to paleontologic calibration points, which are mostly available for relatively old divergences. Nucleotide substitution numbers, however, must be estimated using little-divergent sequences because of mutational saturation. To resolve this methodological problem, we take advantage of the decoupled non-synonymous (i.e. amino acids) and synonymous evolutionary dynamics. We introduced a 3-step method [14], in which we first define groups of sequences showing limited divergences, then estimate the relative species-specific substitution rate at third codon positions within groups, and finally assign a divergence for each of these groups using amino acid sequences. At step 2 and 3, molecular and fossil information are combined thanks to a sophisticated Bayesian method [44].

Using this procedure, we analysed a cytb dataset of 1571 species, which represent $\sim 15 \%$ of the total living species of birds. The average cytb third codon position substitution rate was 0.027 substitution/site/Myr, and the median was 0.018 substitution/site/Myr. These substitution rates are estimated per lineage, per third-codons position. To make them roughly comparable to the popular $2 \%$ of divergence per site per Myr, these numbers must be multiplied by two (to reflect a divergence between two species) and divided by three (assuming that only third codon positions are variable). Doing this would yield an average divergence of $1.8 \%$ per Myr (median: $1.2 \%$ per Myr), which is slightly less than the popular $2 \%$. The distribution across species shows a large variation: the $2.5 \%$ and $97.5 \%$ percentiles are 0.003 and 0.090 substitution/ site/Myr, respectively (Figure 1). Thus, even if we consider the $5 \%$ most extreme substitution rate values as outliers, the cytb third codon position substitution rate shows a thirty-fold range of variation across bird lineages. We quantified the effect of taxonomy on substitution rate variation using a hierarchical ANOVA considering superorder, order, family and genus with random effects. The order level explains $46 \%$ (63\% in simple one-way ANOVA) of the variance in substitution rates across species. Considering only order with more than 20 species, Passerines are the fastest evolving order $(0.038 \pm 0.055$ substitution/site/Myr) and Anseriformes the slowest one $(0.004 \pm 0.0008$ substitution/site/Myr, see Figure 2). Compared with mammals, birds globally evolve 3.7 times slower on average (mammalian average: 0.098 substitution/site/Myr, Nabhloz et al. [14]), and show a range of variation less extreme (coefficient of variation: 1,58 for birds, 1,72 for mammals, Figure 3). 


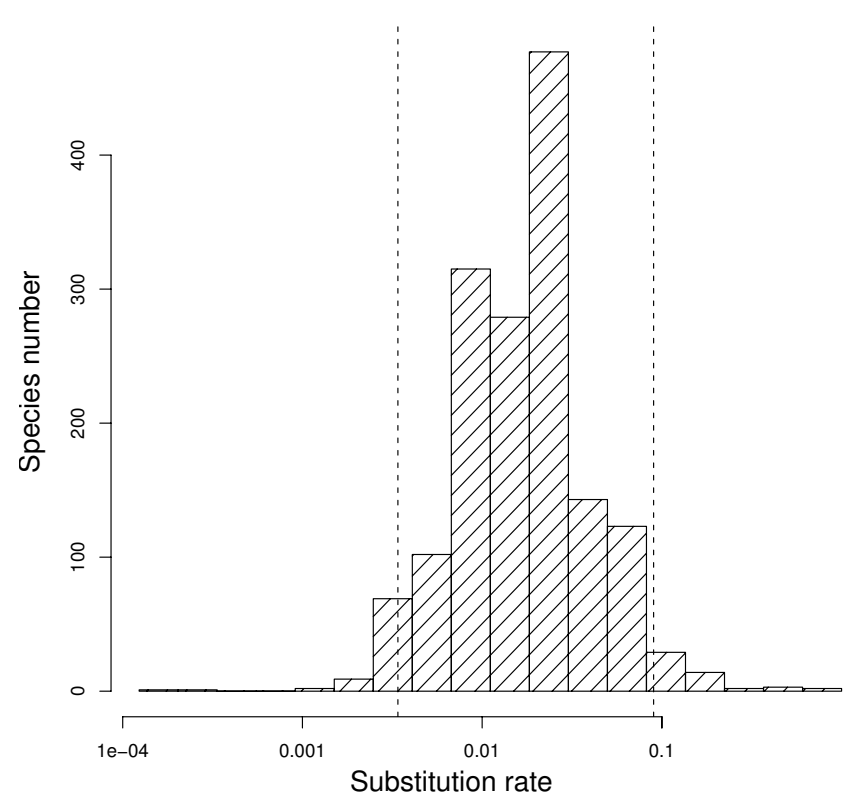

Figure I

Distribution of cytochrome b neutral substitution rate in $\mathbf{1 , 5 6 2}$ bird species. Substitution rates are log transformed and are in unit of substitution per third codon position per million years.

We correlated the species-specific substitution rate to lifehistory traits in order to discriminate between the competing explanatory hypotheses. A smaller amount of life-history data are available in birds than in mammals, especially for female sexual maturity, which is documented in 30 species only. We thus focused on the effects of body mass and maximum longevity using the 196 species in which these two variables are available. In agreement with both the metabolism and the longevity hypotheses, the two life-history traits are negatively linked to the substitution rate. Body mass appears to be a better predictor of the substitution rate $(\mathrm{R} 2=0.39, \mathrm{p}<0.001, \mathrm{n}$ $=196$ ) than maximum longevity, which also shows a strong level of correlation $(\mathrm{R} 2=0.25, \mathrm{p}<0.001, \mathrm{n}=196$, Figure 4, Table 1).

The distribution of (log-transformed) maximum longevity is strongly bimodal in birds, passerines being substantially more short-lived than other birds (see Additional file 1). We separated passerines from non-passerine and re-performed the same analyses in the two subsets separately. The results are shown in Table 2. Surprisingly, the third-codon position substitution rate is positively correlated to body mass and maximum longevity in passerines birds. In non-passerines birds, results are similar to the global analysis: body mass best explains substitution rate variations, and removes the effect of longevity in a twoway ANOVA (Table 2). This result is not consistent with mammals [14].

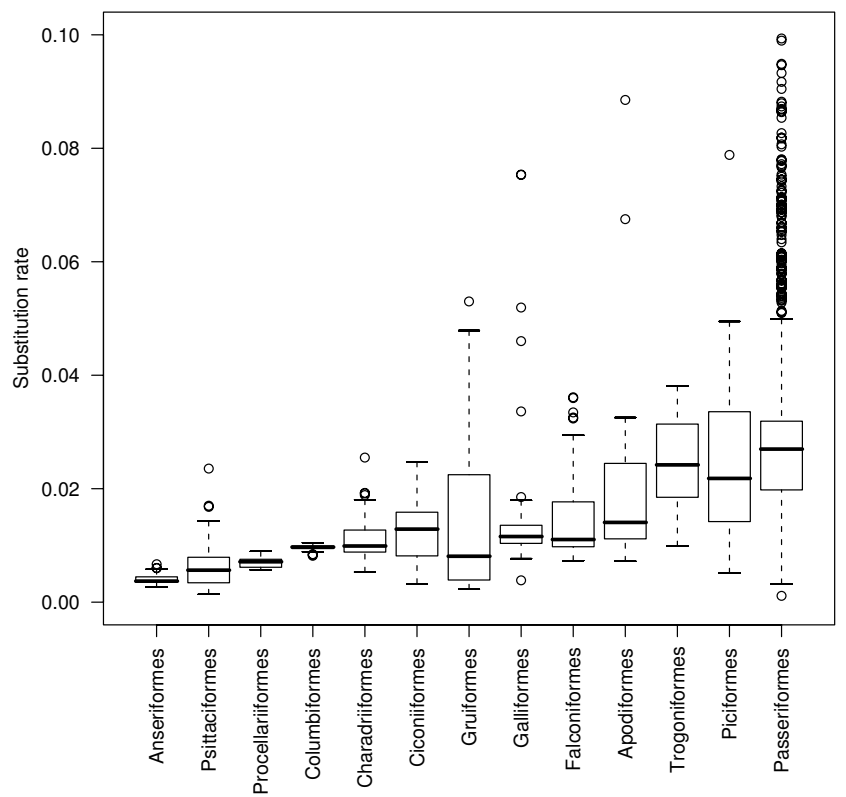

Figure 2

Within-order distribution of the cytochrome $b$ third codon position substitution rate. Substitution rates are in unit of substitution per third codon position per million year. Orders represented by more than 20 species are shown. Values above 0.1 substitution/site/Myrs years are not shown.

In order to check whether these results were robust to the removal of phylogenetic effects, we applied the phylogenetic contrast method [45] to regress phylogeny out of the analyses. In this analysis, we used the chronogramme obtained with amino-acid cytochrome $b$ sequences. The phylogenetic control did not qualitatively change the results although most p-values were decreased (Table 1, Table 2).

Finally, we jointly analysed the mammalian and bird datasets (Table 3, Figure 5), including class (birds vs. mammals) as an explanatory variable. The two-factor model (class + life history trait) revealed that the bird $v$. mammal status has a strong effect on mtDNA substitution rate when body mass is taken into account and a significant but weaker effect as far as longevity is concerned (Table 3). There is, moreover, a significant interaction between body mass and class $(p<0.001)$, but not between longevity and class $(p=0.65)$.

\section{Determination of mtDNA diversity in birds}

Bird cytb polymorphism sequence datasets were retrieved from the Polymorphix database [46]. The synonymous $\left({ }_{s}\right)$ and non-synonymous $\left({ }_{n}\right)$ levels of diversity were computed for the 147 species in which more than four sequences were available. The mean per-site synonymous nucleotide diversity was $0.040 \pm 0.041$. The most variable 


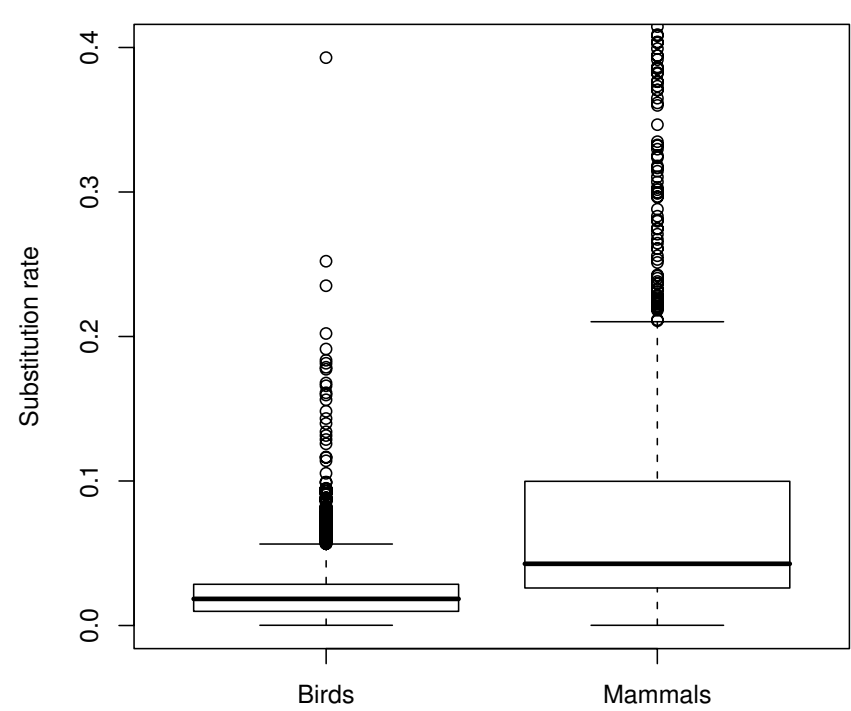

Figure 3

Distribution of cytochrome b neutral substitution rate in 1,696 mammalian species (Data from [14]) and $I, 57$ I birds species (this study). Substitution rates are in unit of substitution per third codon position per million years. Values above 0.4 substitution/site/Myrs years are not shown.

species was Shelley's Greenbul (Andropadus masukuensis, Pycnonotidae, Passeriformes, $s=0.170)$, and two species showed no synonymous variations (Arabian Bustard, Ardeotis arabs, Otididae, Gruiformes, and Snowy owl, Bubo scandiacus, Strigidae, Strigiformes).

To test the relationship between population size and mtDNA diversity, we first correlated nucleotide polymorphism and body mass, taken as a proxy of population size,
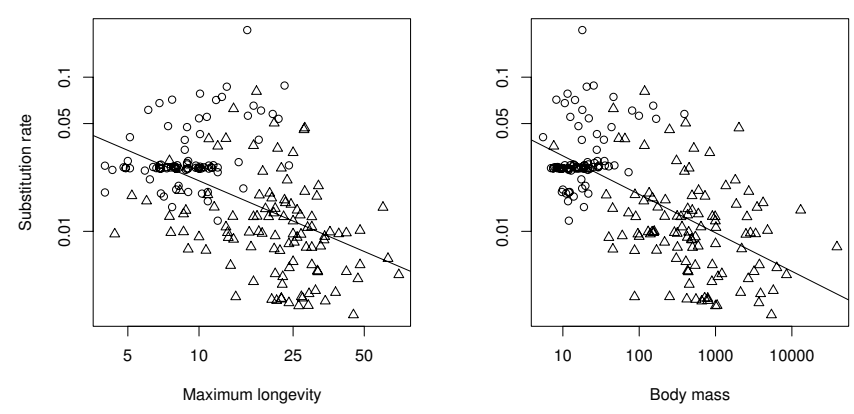

Figure 4

Relationship between substitution rate and life-history traits. a) Relationship with body mass (log transformed and in grams), b) Relationship with maximum longevity (log transformed and in years). Circles are for passerine birds ( $n$ $=88)$, triangles for non-passerine birds $(n=108)$. Substitution rates are log transformed and are in unit of substitution per third codon position per million years.
Table I: Effects of life-history variables on mtDNA substitution rate in bird species without $(A)$ and with phylogenetic control (B).

\begin{tabular}{|c|c|c|c|c|}
\hline Model & slope & $\mathrm{R}^{2}$ & pl & p2 \\
\hline \multicolumn{5}{|l|}{ (A) Without Phylogenetic control } \\
\hline Body mass & -0.25 & 0.39 & $<0.01$ & \\
\hline Maximum longevity & -0.66 & 0.25 & $<0.01$ & \\
\hline Body mass + Maximum longevity & & 0.39 & $<0.01$ & 0.42 \\
\hline \multicolumn{5}{|l|}{ (B) With Phylogenetic control } \\
\hline Body mass & -0.22 & 0.38 & $<0.01$ & \\
\hline Maximum longevity & -0.46 & 0.19 & $<0.01$ & \\
\hline Body mass + Maximum longevity & & 0.38 & $<0.01$ & 0.89 \\
\hline
\end{tabular}

as in Nabholz et al. [18] and Berlin et al. [24]. ${ }_{s}$ and body mass were negatively correlated but, contrary to Berlin et al. [24], this relationship was not significant $\left(\mathrm{R}^{2}=0.019\right.$, $p=0.30, \mathrm{n}=56$ ). Secondly, we applied a more direct analysis using population size estimates for North American breeding birds through the North American Landbird Conservation Plan [22]. Such data were available for 28 strictly North American species of our data set. We found no correlation between direct population size estimate and cytb synonymous diversity (Figure 6, $\mathrm{t}=0.031, p=$ 0.81 , Kendall's test). These results confirm those obtained in mammals [18], here using direct estimations of population sizes instead of life-history and ecological proxies. The dataset, however, is much smaller in the present analysis.

We tested the taxonomic effect on ${ }_{s}$ using one-way ANOVAs on order and family level. We found that neither the family nor the order level had a significant effect. The sole taxonomic variable having a significant effect on mtDNA diversity is the passerine/non-passerine status, passerines being more diverse than other birds (passerines: $\mathrm{n}=79$, non-passerines: $\left.\mathrm{n}=68, \mathrm{R}^{2}=0.03, \mathrm{p}<0.05\right)$.

We compared the distribution of ${ }_{s}$ between birds and mammals. Similarly to Berlin et al. [24], we found that birds have a lower average synonymous diversity than mammals $\left(\mathrm{n}=169, \mathrm{R}^{2}=0.09, \mathrm{p}<0.001\right.$, Additional file $2 \mathrm{a})$, but this difference is largely reduced when we controlled for the mutation rate (previously estimated above after control, $\mathrm{R}^{2}=0.03, \mathrm{p}=0.032$, Additional file $2 \mathrm{~b}$ ). Interestingly, we detected no significant difference of allozyme heterozygosity between mammals (average $\mathrm{H}=$ $0.046 \pm 0.033)$ and birds $(0.053 \pm 0.028)$ using the Nevo et al. [47] data set (birds: $\mathrm{n}=42$, mammals: $\mathrm{n}=164, \mathrm{R}^{2}=$ $0.01, \mathrm{p}=0.08)$. As in mammals, we found a positive and significant correlation between species-specific cytb thirdcodon substitution rate and cytb synonymous diversity per genus $\left(n=46, R^{2}=0.10, p=0.02\right.$, Figure 7$)$. These analyses confirm that the mutation rate is a major deter- 
Table 2: Effects of life-history variables on $m$ tDNA substitution rate in passerines $(n=88)$ vs. other bird species $(n=108)$ without $(A)$ and with phylogenetic control (B).

\begin{tabular}{|c|c|c|c|c|c|c|c|c|}
\hline \multirow[b]{2}{*}{ Model } & \multicolumn{4}{|c|}{ Passerines } & \multicolumn{4}{|c|}{ Non-passerines } \\
\hline & slope & $\mathrm{R}^{2}$ & pl & $p 2$ & slope & $\mathrm{R}^{2}$ & pl & $p 2$ \\
\hline \multicolumn{9}{|l|}{ (A) Without Phylogenetic control } \\
\hline Body mass & 0.22 & 0.11 & $<0.01$ & & -0.17 & 0.12 & $<0.01$ & \\
\hline Maximum longevity & 0.48 & 0.15 & $<0.01$ & & -0.41 & 0.09 & $<0.01$ & \\
\hline Body mass + Maximum longevity & & 0.17 & 0.14 & 0.02 & & 0.13 & 0.01 & 0.13 \\
\hline \multicolumn{9}{|l|}{ (B) With Phylogenetic control } \\
\hline Body mass & 0.22 & 0.12 & $<0.01$ & & -0.11 & 0.05 & 0.02 & \\
\hline Maximum longevity & 0.4 & 0.11 & $<0.01$ & & -0.28 & 0.05 & 0.03 & \\
\hline Body mass + Maximum longevity & & 0.16 & 0.04 & 0.05 & & 0.06 & 0.17 & 0.22 \\
\hline
\end{tabular}

minant of mtDNA polymorphism in birds as well as in mammals.

Finally, we compared the ratio of non-synonymous to synonymous changes within $\left(P_{n} / P_{s}\right)$ and between species $\left(D_{n} / D_{s}\right)$ in birds and mammals - these ratio were computed when an outgroup was available. The two groups showed a similar $D_{n} / D_{s}$ (birds: $\mathrm{n}=81$, average $D_{n} / D_{s}=$ $0.019 \pm 0.011 ;$ mammals: $\mathrm{n}=76$, average $D_{n} / D_{s}=0.023 \pm$ $\left.0.024 ; \mathrm{R}^{2}=0.01, \mathrm{p}=0.11\right)$, but the $P_{n} / P_{s}$ ratio was higher in birds than in mammals (birds: average $P_{n} / P_{s}=0.118 \pm$ 0.110 ; mammals: average $P_{n} / P_{s}=0.076 \pm 0.055 ; \mathrm{R}^{2}=0.05$, $\mathrm{p}<0.01$ ), as previously reported by Berlin et al. [24].

\section{Discussion}

mtDNA substitution rates - support for the longevity hypothesis

We showed that the mtDNA mutation rate, measured through the neutrally-evolving third codon positions of
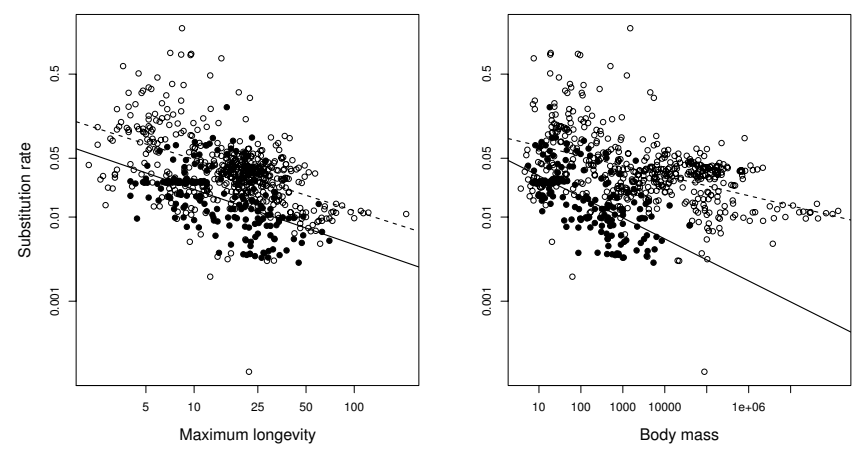

Figure 5

Relationship between substitution rate and life-history traits. a) Body mass (log transformed and in grams), b) Maximum longevity (log transformed and in years). Open circles and dotted regression line are for mammals $(n=500)$, close circles and solid regression line for birds $(n=196)$. Substitution rates are log transformed and are in unit of substitution per third codon position per million years. cytb, is highly variable between bird lineages. The molecular clock hypothesis does not apply to bird mtDNA evolution, fast-evolving species being thirty times more rapid than slow-evolving ones. The method we applied is particularly prone to reveal the actual amplitude of substitution rate variation: by overcoming the problem of mutational saturation, it allowed us to use an extensive dataset encompassing the whole taxonomic diversity of birds. This study, therefore, provides a synthetic view of substitution rate variations across bird lineages, bringing an important result in the field of molecular dating, in which the rough approximation of $2 \%$ of substitution per million of years is still debated $[41,42,40,43,39]$. Our analysis suggests that the molecular clock assumption should be avoided as far as bird mtDNA is concerned. The conclusions of Pereira and Baker [43] (obtained from fullgenome data), and some studies done at smaller scale (reviewed in [39]) are here confirmed by cytochrome $b$ third codon positions at wide taxonomic scale. Although the average rate in birds is close to this value, the $2 \%$ per site per million year calibration is a very bad summary of the whole picture: the rate is essentially (up to five times) higher than $2 \%$ in passerines, and (up to ten times) lower in non-passerines (Figure 1). Users of mtDNA as a tools for inferring divergence dates should imperatively use statistical phylogenetic methods accounting for substitution rate variation across lineages, the so-called clock-relaxed methods [44,48-50].

Several interesting results were revealed thanks to the comparison with mammals. First, birds show a more narrow range of variation of life-history traits than mammals. For example body mass varies from $5.5 \mathrm{~g}$ to $3.9 \mathrm{~kg}$ in the bird dataset, whereas it varies from $3.7 \mathrm{~g}$ to 138 tones in mammals. This result confirms the general influence of life-history traits on neutral mtDNA substitution rate in birds and mammals. This result is important because such a relationship was not reported in every taxonomic group [51], and even not by some studies in mammals [52,53]. Secondly, the neutral mtDNA substitution rate is lower in birds than in mammals, and this difference increases if 
Table 3: Effects of life-history variables and bird $(n=196)$ vs. mammal $(n=500)$ status (referred as Class) on mtDNA substitution rate.

\begin{tabular}{|c|c|c|c|c|}
\hline Model & Variable & Effect & t value & $P$ \\
\hline \multicolumn{5}{|c|}{ (A) Body mass + Class } \\
\hline & Body mass & -0.25 & -8.9 & $<0.01$ \\
\hline & Class (Mammals) & 0.22 & 3.1 & $<0.01$ \\
\hline & Body mass: Class (Mammals) & 0.13 & 4.4 & $<0.01$ \\
\hline \multicolumn{5}{|c|}{ (B) Maximum longevity + Class } \\
\hline & Maximum longevity & -0.65 & -9.4 & $<0.01$ \\
\hline & Class (Mammals) & 0.31 & 2.5 & 0.01 \\
\hline & Maximum longevity: Class (Mammals) & 0.04 & 0.5 & 0.65 \\
\hline
\end{tabular}

body mass is regressed out of the analysis (Table 2). For example, birds evolve four times slower than mammals as far as small species (body mass $<500 \mathrm{~g}$ ) are concerned. This result is in agreement with the longevity hypothesis, but not with the metabolic rate hypothesis. According to the latter model, birds should show a higher average mutation rate than mammals, because of their higher mass-specific metabolic rate and their lower average body mass [54].

During the course of evolution, birds have acquired adaptations to manage their high mass-specific metabolic rate, including an increased of reactive oxygen species (ROS) protection and lower ROS production $[55,56]$. In the context of the mitochondrial theory of aging, which postulates that ROS production as a byproduct of mitochondrial respiration contributes to aging, these

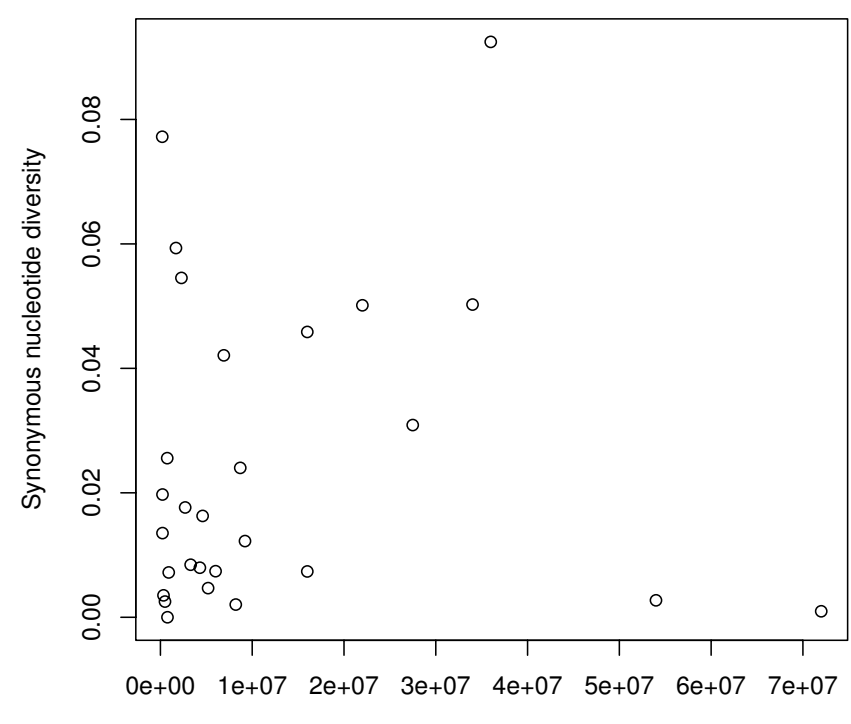

Population size estimation

\section{Figure 6}

mtDNA synonymous diversity $(\mathrm{s})$ vs. direct population size estimate in birds $(\mathbf{n}=\mathbf{2 8})$. mtDNA synonymous diversity $\left({ }_{s}\right)$ vs. direct population size estimate in birds $(\mathrm{n}=$ 28). adaptations could be interpreted as a response to the elevated longevity of birds [57]. The reduced rate of ROS production could explain the lower average mutation rate of birds mtDNA, as compared to mammals (see also [26]). It is surprising, therefore, to find a predominant influence of body mass, not longevity, in the within-birds analysis. Passerines show a positive relationship between longevity (or body mass) and substitution rate, which is quite surprising. We have no valid explanation for this relationship so far, perhaps because it is not biologically relevant - this relationship is the single result that was not recovered when we used other methods of substitution rate estimation (see Additional file 3). Within non-passerines, which are essentially long-lived species, body mass is still the variable best explaining substitution rate variations

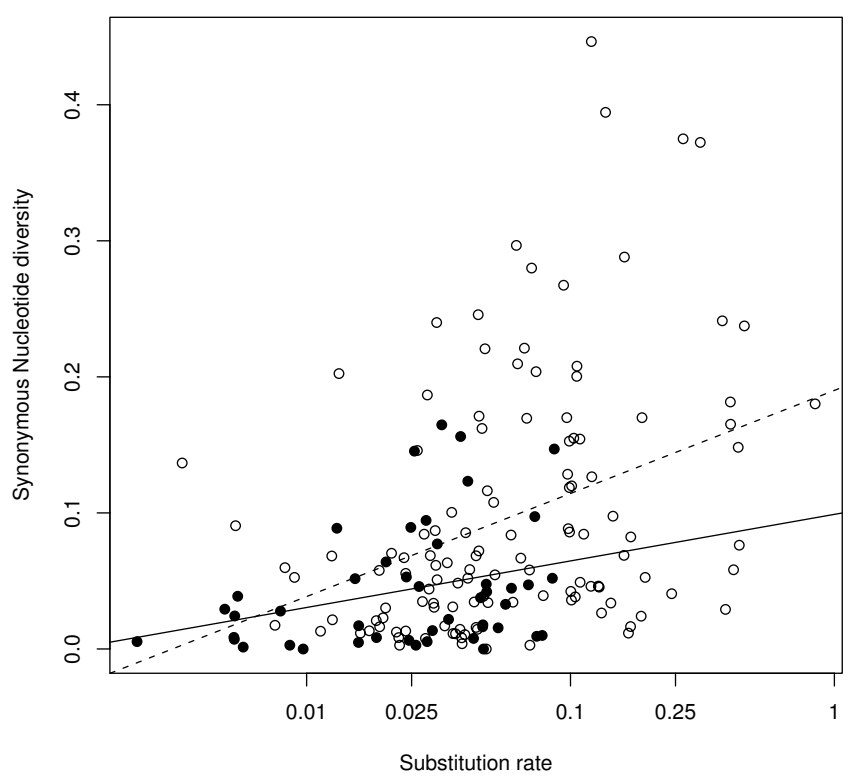

Figure 7

mtDNA synonymous diversity $\left({ }_{s}\right)$ and substitution rate. Open circles and dotted regression line are for mammals $(n=123)$, close circles and solid regression line for birds $(n=46)$. Substitution rates are in log-transformed and are in unit of substitution per third codon position per million years. 
(Table 2). Using DNA-DNA hybridization data (and a smaller dataset), Mooers and Harvey [32] found no support for the metabolic rate hypothesis, but reported a significant generation time effect. In this context, it would be interesting to enlarge the female sexual maturity data set, in order to test the generation time hypothesis with our extended mitochondrial dataset.

\section{Determination of mtDNA diversity in birds}

The neutral genetic diversity primarily depends on effective population size and mutation rate. Because population sizes appear so variable in time and between species, variations in genetic diversity across species have been frequently interpreted in demographic terms, e.g. [58,59]. Recently, the effect of population size on mtDNA diversity has been challenged $[18,20]$. The large variations in mutation rates we report in birds suggest that mutational effects should be carefully taken into account in studying mtDNA polymorphism in this group, as well as in mammals [18].

In general, the relationship between population size and mtDNA diversity is difficult to evaluate because of the scarcity of population size estimates for wild species. Lifehistory traits, like body mass, have therefore been used as proxy of population size $[18,60]$. Birds are an exception: thanks to the broad interest of the scientific community in bird ecology and systematics, direct estimates of species effective sizes are available for North American species [22]. We correlated these population size estimates to synonymous mtDNA diversity and found no significant relationship. The data set is rather small $(n=28)$, but we note that this relationship should theoretically be strong, given the wide range of population sizes (2 orders of magnitude). When using body mass as an indicator of population size, thus increasing much the data set, we found a negative correlation with cytb synonymous diversity, but this result was not significant. Berlin et al. [24] report a significant relationship between ${ }_{s}$ and body mass in birds using genus averages. If we apply the same method, we also find a significant relationship $\left(\mathrm{n}=37, \mathrm{R}^{2}=0.16, p=\right.$ 0.01 ), but the effect is removed when the passerine/nonpasserine status is taken into account (multiple regression, $p$ body mass $=0.25$ ). This result is in agreement with our previous mammalian analysis, in which the weak effect of body mass was removed by a taxonomic control [18]. So similarly to mammals, the mitochondrial genetic diversity is essentially uncorrelated to life-history traits in birds, and when it is so, it is most likely via the influence of mutation rate, not population size.

\section{Mitochondrial selective regime in birds}

To explain the lack of relationship between mtDNA diversity and proxies of population size in mammals, Nabholz et al. [18] invoked demographic stochasticity, while Bazin et al. [20] invoked recurrent hitchhiking effects for other species having larger expected population sizes (invertebrates and marine species). What about Birds? To address this question we computed the neutrality index (NI $=P_{n} /$ $P_{s} / D_{n} / D_{s} ;[61]$, see Methods). NI $<1$ indicates positive selection, while NI > 1 indicates purifying selection. The average NI in birds is significantly higher than in mammals $\left(\mathrm{R}^{2}=0.058, \mathrm{p}<0.01\right.$, Additional file 2$)$ and only three species of birds show a NI $<1$ (seven in mammals). The high NI values in birds are due to their high $P_{n} / P_{s}$ ratios $-D_{n} / D_{s}$ ratios are similar to those of mammals. This result indicates that mtDNA evolution in birds is mainly governed by purifying selection, as previously reported [24]. It is also indicative of a strong level of constraint acting on bird cytochrome $b$ sequences, which is known as the avian constraint hypothesis [62].

Like mammals, birds appear to have population sizes low enough to belong to the "drift domain", rather than the "draft domain" sensu Gillespie [63] (see discussion in [18]). In the absence of a significant impact of positive selection, we have to invoke strong demographic stochasticity to explain the lack of relationship between population size and mtDNA diversity. Mutation rate is expected to be a major determinant of within-species genetic diversity whether populations are at mutation-drift equilibrium (standard theory) or not $[64,65]$. Consistently, we report a significantly positive relationship between cytb diversity and substitution rate (Figure 7), again paralleling the mammalian situation.

In a recent paper, Berlin et al. [24] proposed an alternative hypothesis to explain mtDNA polymorphism patterns in birds. They suggested that Hill-Robertson effects due to complete association with the $\mathrm{W}$ chromosome could explain the lower synonymous mitochondrial diversity and the higher $P_{n} / P_{s}$ observed in birds, as compared to mammals. This should also explain the lack of correlation between neutral polymorphism and population size. Outwardly, we confirm this hypothesis by showing that the lower synonymous mitochondrial diversity is not found for nuclear allozymes. But this picture is complicated by the globally lower mtDNA substitution rate in birds than in mammals. Controlling for mutation rates actually removes the major part of the difference between birds and mammals. To quantify the effect of mutation rate variation on synonymous mtDNA diversity we combined the bird and mammal polymorphism and neutral substitution rate datasets and performed a multiple regression of substitution rate and bird/mammal status (class) on synonymous mtDNA diversity. This model explains $21 \%$ of s variation (birds: $\mathrm{n}=46$, mammals: $\mathrm{n}=123, p<0.001$ ). The result also shows that the mutation rate has a strong effect on ${ }_{s}(\mathrm{t}=4.9 ; p<0.001)$ and considerably reduces the effect of class $(\mathrm{t}=2.1, p=0.021)$. This analysis indicates that a subtential part of the difference between birds and mammalian ${ }_{s}$ is explained by their distinct mutation 
rates (see Additional file $4 \mathrm{~b}$ ). The difference in mutation rates, however, cannot explain the higher level of mitochondrial $P_{n} / P_{s}$ found in birds. Birds and mammals have apparently comparable effective population sizes, as suggested by their similar average allozymic heterozygosity. The higher $P_{n} / P_{s}$ ratio in birds may therefore be due to Hill-Robertson interferences between the mitochondrial and $\mathrm{W}$ chromosomes, as suggested by Berlin et al. [24], although this effect has only a weak influence on absolute levels of ${ }_{s^{\prime}}$ when mutation rate is controlled for.

\section{Conclusion}

In this work, we achieved a comprehensive comparison of mitochondrial substitution rate variation and mitochondrial diversity between birds and mammals. Cytb neutral substitution rates are, on average, 3.9 times slower in birds than in mammals. Among-lineages variability, although substantial, is also lower in birds than in mammals. This analysis corroborates the longevity hypothesis about mutation rate determination $[14,17]$. Despite their higher metabolic rate, birds undergo a lower mitochondrial mutation rate, probably because of the beneficial effect of reduced oxidative damage in long-lived species $[26,28]$. We suggest that the low mtDNA mutation rate of birds might explain their exceptional mass-specific longevity.

As for mammals, we report no evidence of adaptive selection on cytb sequence evolution, but still no population size effect, even when using direct estimates of population size available for North American species. We thus invoke the same demographic instability explanation as previously proposed for mammals [18], although selective interferences with the $\mathrm{W}$ chromosome [24] could contribute to the noisy relationship between mtDNA diversity and population size. We also confirm the importance of mutation rate as a determinant of mtDNA diversity - the lower mutation rates in birds almost fully explain their lower synonymous diversity.

This study confirms and extends the message of caution expressed by Nabholz et al. $[18,14]$ about the usage of mtDNA as a molecular marker of biodiversity in vertebrates: (i) mtDNA diversity is not related to species abundance; (ii) mtDNA greatly departs the molecular clock hypothesis. The $2 \%$ per site per million year calibration (estimated from primate data) has no degree of generality, and should not be used for dating purposes in the absence of fossil data.

\section{Methods}

\section{Sequence data for substitution rate analysis}

Complete bird cytochrome $b$ sequences were extracted from National Center for Biotechnology information/ Genbank. One sequence per species (the longest, excluding indeterminations) was selected.
Accession numbers are given in Additional file 5. The 5'most 102 nucleotides were removed from the alignments, because missing in a large number of species. Total alignment length was 1043 nucleotides.

\section{Sequence data and Allozyme data for polymorphism analysis}

An mtDNA dataset was built from Polymorphix [46]. Polymorphix is a database dedicated to sequence polymorphism. It contains within-species homologous sequence families built from EMBL/GenBank under suitable similarity and bibliographic criteria. To obtain a homogeneous dataset, comparable with a previous mammalian study [18], only the protein coding cytochrome $b$ gene was used. We extracted from Polymorphix every bird sequence family for which any $>100$-bases-long cytochrome $b$ fragment was available in four individuals or more. Polymorphix sequence families were aligned using CLUSTALW [66], inspected by eye and corrected when required. Dubious sequences (badly aligned or including many undetermined nucleotides) were manually removed. Alignments are available at http://kimura.univmontp2.fr/ benoit/data.html.

Estimates of allozyme heterozygosity in 74 bird and 194 mammalian species were obtained from [47]. Allozyme heterozygosities were averaged using 10-30 loci. The most popular allozyme loci are shared by many species. Allozyme heterozygosities are therefore fairly comparable between species.

\section{Substitution Rate estimation}

We used the same uncoupled method as previously developed for mammals [14]. The whole cytochrome $b$ data set was split in groups within which sequence divergence is moderate. To achieve this, the GenBank taxonomic classification was traversed recursively, starting from birds orders and moving toward lower levels. For each traversed taxonomic group, we 1) gathered the corresponding third codon position sequences, 2) aligned sequences using ClustalW [66], 3) built a maximum likelihood phylogenetic tree using PHYML [67], general time reversible + Gamma model of nucleotide evolution, and 4) calculated the pairwise patristic distances for every pair of species (defined as the sum of branch lengths in the path connecting the 2 species in the tree). When the median pairwise distance between species was lower than 0.4, the current taxonomic group was selected for further analysis, (except for the Falconiformes and Ciconiiformes orders, for which monophyly is uncertain $[68,69])$, and the taxonomic traversal was stopped. Otherwise, the above procedure was applied to underlying taxonomic groups. The within group, species-specific relative neutral substitution rates were estimated using two different softwares: MULTIDIVTIME [44] and MCMCTREE [50,70] available 
in the PAML packages [71]. The major difference is that MULTIDIVTIME makes use of a normal approximation of the likelihood, and only implements the F84 $+\Gamma$ model of sequence evolution [44], whereas MCMCTREE performs exact likelihood calculation and can be used with different models of sequence evolution - we used the HKY $+\Gamma$ The Monte Carlo Markov Chain was run for 1 million generations after a burn-in of 200,000 generations that achieved stationarity.

Bird phylogeny is still partly uncertain, particularly the basal relationships within the Neoaves clade $[68,69,72$ $74]$. We used two alternative phylogenetic trees to date divergences. The first one is conform to Ericson et al. [68] topology, obtained using mostly nuclear markers, and the second one is conform to the complete genome mitochondrial topology proposed by Slack et al. [69]. In both topologies, the relationships within passerines were made congruent to Barker et al. [75]. Groups were dated by applying MULTIDIVTIME to amino-acid sequences (two representative species per group, model mtREV $+G)$. Twelve fossil calibrations points were used (Additional file 6). The results were very similar whatever the method and the tree (Additional files 3 and 7), so we chose to present only the results obtained with MULTIDIVTIME using the mitochondrial genome topology, except when mentioned. We checked that neither the mean nor the variance of species-specific estimated substitution rates are correlated to terminal branch lengths (not shown). Sequence management and GenBank taxonomic exploration were achieved using homemade $\mathrm{C}++$ programs based on the Bio++ libraries [76].

\section{Polymorphism sequence data analyses}

Two measures of molecular genetic diversity were used, namely the nucleotide diversity [77] and Watterson's statistics ${ }_{w}$ [78]. In the case of the haploid, maternally transmitted mtDNA, both statistics are unbiased estimates of the $N$ ef product under the assumption of neutrality and mutation/drift equilibrium, where $N$ ef is the effective population size of females and the locus mutation rate. and ${ }_{w}$ were calculated from the total length of the analysed fragments, and expressed in per-site level of diversity (after being divided by sequence length). We also measured the synonymous and non-synonymous nucleotide diversity $\left({ }_{s} / n\right)$, also expressed per synonymous site and per non-synonymous site.

The Neutrality Index (NI, [61]) was calculated for dataset 1 when outgroups were available. This index aims at comparing the ratio of non-synonymous (= amino acid changing) to synonymous (silent) changes within species $(n / s)$ and between species $\left(D_{n} / D_{s}\right)$ : NI is one when evolution is neutral, higher than one under purifying selection, and lower than one in case of adaptation. ${ }_{n}$ and ${ }_{s}$ were estimated as described above. $D_{n}$ and $D_{s}$ are the non-synony- mous and synonymous pairwise sequence divergences between related taxa, calculated using the maximum likelihood method implemented in codeml in PAML 4 [71]. Excluding low frequency variants $(<0.125)$ when estimating NI yielded similar results (not shown). The phylogenetic tree was obtain using PHYML software [67] with $\mathrm{TN} 93+\Gamma$ model of sequences evolution. We used the maximum likelihood method because of the potential underestimation of $D_{s}$ in simple pairwise distance due to multiple hits. Alignments are available at http:// kimura.univ-montp2.fr/ benoit/data.html.

\section{Life History data and Population size estimation}

Body mass, age of female sexual maturity, and maximum longevity were obtained from the AnAge database [79]. Direct estimates of population size are available for North American breeding birds in the North American Landbird Conservation Plan, Breeding Bird Survey abundance data [22], http://www.partnersinflight.org/. Life history data and rate estimates are available at http://kimura.univmontp2.fr/ benoit/data.html.

\section{Statistical analyses}

Genetic diversity measures were arcsine-transformed [80] and analyzed under the general linear model assumptions using R [81]. Quantitative life-history variables were logtransformed. One-way and two-way ANOVA with interactions were performed on transformed variables. Fisher's tests were performed using class II errors.

\section{Authors' contributions}

All authors designed research project. BN compiled the data and performed the analyses. All authors wrote and approved the final manuscript.

\section{Additional material}

\section{Additional file 1}

Figure S1. Distribution of bird maximum longevity. The maximum longevity is log transformed and is in years. Black: whole dataset; shading yellow: passerines birds $(n=88)$; shading blue: no passerines birds $(n=108)$ Click here for file

[http://www.biomedcentral.com/content/supplementary/14712148-9-54-S1.pdf]

\section{Additional file 2}

Figure S2. Neutrality index (NI) distribution in birds $(n=81)$ and mammals $(n=75)$. NI values greater than 20 were forced to 20 for clarity.

Click here for file

[http://www.biomedcentral.com/content/supplementary/14712148-9-54-S2.pdf] 


\section{Additional file 3 \\ Table S4. Effects of life-history variables on mtDNA substitution rate in passerines versus other bird species according the different combination of topologies and programs. \\ Click here for file \\ [http://www.biomedcentral.com/content/supplementary/1471- \\ 2148-9-54-S3.pdf] \\ Additional file 4 \\ Figure S3. a) mtDNA synonymous diversity $\left(\pi_{\mathrm{s}}\right)$ in birds $(n=46)$ and mammals $(n=123)$. The median, quartiles, and extrema of the distribu- tion of $\pi_{\mathrm{s}}$ are given. $b$ ) Residuals of the relationship between mtDNA syn- onymous diversity $\left(\pi_{\mathrm{s}}\right)$ and third-codon position substitution rate in birds and mammals. \\ Click here for file \\ [http://www.biomedcentral.com/content/supplementary/1471- 2148-9-54-S4.pdf]}

\section{Additional file 5}

Table S2. GenBank accession numbers, taxonomy, mitochondrial substitution rate and life-history traits of the 1571 species used in the substitution rate analysis.

Click here for file

[http://www.biomedcentral.com/content/supplementary/14712148-9-54-S5.csv]

\section{Additional file 6}

Table S1. Fossil calibration dates used in this study (in million years). Click here for file

[http://www.biomedcentral.com/content/supplementary/14712148-9-54-S6.pdf]

\section{Additional file 7}

Table S3. Effects of life-history variables on $m t D N A$ substitution rate in bird species according the different combination of topologies and programs.

Click here for file

[http://www.biomedcentral.com/content/supplementary/1471-

2148-9-54-S7.pdf]

\section{Acknowledgements}

This work was supported by the Agence National de la Recherche (projet MITOSYS) and the Centre National de la Recherche Scientifique. We thank the three anonymous referees for their helpful comments. We thank the ISEM bioinformatic platform for providing the computing resources. This is manuscript number ISEM 2009-017.

\section{References}

I. Ballard J, Whitlock M: The incomplete natural history of mitochondria. Molecular Ecology 2004, I 3(4):729-744.

2. Lane N: Power, Sex, Suicide. Mitochondria and the Meaning of Life Oxford University Press; 2005.

3. Brown WM, George M, Wilson AC: Rapid evolution of animal mitochondrial DNA. Proceedings of the National Academy of Sciences of the United States of America 1979, 76(4): 1967-1971.

4. Lynch M: The origins of eukaryotic gene structure. Molecular Biology and Evolution 2006, 23(2):450-468.

5. Rand DM: Thermal habit, metabolic-rate and the evolution of mitochondrial-DNA. Trends in Ecology \& Evolution 1994, 9(4): $|25-| 3 \mid$.

6. Palmer JD, Adams KL, Cho YR, Parkinson CL, Qiu YL, Song KM: Dynamic evolution of plant mitochondrial genomes: mobile genes and introns and highly variable mutation rates. Proceedings of the National Academy of Sciences of the United States of America 2000, 97(13):6960-6966.

7. Hellberg ME: No variation and low synonymous substitution rates in coral $\mathbf{m t d n a}$ despite high nuclear variation. BMC Evolutionary Biology 2006, 6:24-24.

8. Huang D, Meier R, Todd PA, Chou LM: Slow mitochondrial COI sequence evolution at the base of the metazoan tree and its implications for DNA barcoding. Journal of Molecular Evolution 2008, 66(2): 167-174.

9. Springer M, Debry R, Douady C, Amrine H, Madsen O, de Jong W, Stanhope M: Mitochondrial versus nuclear gene sequences in deep-level mammalian phylogeny reconstruction. Molecular Biology and Evolution 200I, I8(2): I 32-143.

10. Galewski T, Tilak M, Sanchez S, Chevret P, Paradis E, Douzery EJP: The evolutionary radiation of arvicolinae rodents (voles and lemmings): relative contribution of nuclear and mitochondrial dna phylogenies. BMC Evolutionary Biology 2006, 6:80-80.

II. Ratnasingham S, Hebert PDN: BOLD: the barcode of life data system. Molecular Ecology Notes 2007, 7(3355-364 [http://www.Bar codinglife. Org].

12. Springer MS, Amrine HM, Burk A, Stanhope MJ: Additional support for Afrotheria and Paenungulata, the performance of mitochondrial versus nuclear genes, and the impact of data partitions with heterogeneous base composition. Systematic Biology 1999, 48:65-75.

13. Galtier N, Enard D, Radondy Y, Bazin E, Belkhir K: Mutation hot spots in mammalian mitochondrial DNA. Genome Research 2006, 16(2):215-222

14. Nabholz B, Glémin S, Galtier N: Strong variations of mitochondrial mutation rate across mammals-the longevity hypothesis. Molecular Biology and Evolution 2008, 25:120-130.

15. Samuels D: Mitochondrial DNA repeats constrain the life span of mammals. Trends in Genetics 2004, 20(5):226-229.

16. Khaidakov M, Heflich RH, Manjanatha MG, Myers MB, Aidoo A: Accumulation of point mutations in mitochondrial DNA of aging mice. Mutation Research/dna Repair 2003, 526(I-2): I-7.

17. Welch JJ, Bininda-Emonds ORP, Bromham L: Correlates of substitution rate variation in mammalian protein-coding sequences. BMC Evolutionary Biology 2008, 8:53-53.

18. Nabholz B, Mauffrey JF, Bazin E, Galtier N, Glémin S: Determination of mitochondrial genetic diversity in mammals. Genetics 2008, I78:35I-36I.

19. Mulligan C, Kitchen A, Miyamoto M: Comment on "population size does not influence mitochondrial genetic diversity in animals". Science 2006, 3 I 4(5804): I390-1390.

20. Bazin E, Glemin S, Galtier N: Population size does not influence mitochondrial genetic diversity in animals. Science 2006, 3 I 2(5773):570-572.

21. Hurst G, Jiggins F: Problems with mitochondrial DNA as a marker in population, phylogeographic and phylogenetic studies: the effects of inherited symbionts. Proceedings Of The Royal Society B-biological Sciences 2005, 272(I 572): I525-I534.

22. Rich T, Beardmore C, Blancher P, Bradstreet M, Butcher G, Demarest D, Dunn E, Hunter W, Iñigo Elias E, Kennedy J, Martell A, Panjabi A, Pashley D, Rosenberg K, Rustay C, Wendt J, Will T: Partners in Flight North American landbird conservation plan Cornell Lab of Ornithology; 2004.

23. Berlin S, Ellegren $\mathrm{H}$ : Evolutionary genetics. Clonal inheritance of avian mitochondrial DNA. Nature 200I, 4I3(685I):37-38.

24. Berlin S, Tomaras D, Charlesworth $B$ : Low mitochondrial variability in birds may indicate Hill-Robertson effects on the W chromosome. Heredity 2007, 99(4):389-396.

25. Marais GAB: The Hill-Robertson effects extend from nucleus to mitochondria. Heredity 2007, 99(4):357-358.

26. Hickey AJR: Avian mtDNA diversity: An alternate explanation for low mtDNA diversity in birds: an age-old solution? Heredity 2008, 100(5):443-443.

27. Lane N: Mitochondria and the $\mathbf{W}$ chromosome: low variability on the $\mathbf{W}$ chromosome in birds is more likely to indicate selection on mitochondrial genes. Heredity 2008, I 00(5):444-445.

28. Holmes DJ, Flückiger R, Austad SN: Comparative biology of aging in birds: an update. Experimental Gerontology 2001, 36(46):869-883. 
29. Holmes DJ, Ottinger MA: Birds as long-lived animal models for the study of aging. Experimental Gerontology 2003, 38(I II 2): I365-1375.

30. $\mathrm{Li}$ WH, Tanimura M, Sharp PM: An evaluation of the molecular clock hypothesis using mammalian DNA sequences. Journal of Molecular Evolution 1987, 25(4):330-342.

31. Ohta T: An examination of the generation-time effect on molecular evolution. Proceedings of the National Academy of Sciences of the United States of America 1993, 90(22): I0676-10680.

32. Mooers $\mathrm{AO}$, Harvey $\mathrm{PH}$ : Metabolic rate, generation time, and the rate of molecular evolution in birds. Molecular Phylogenetics and Evolution 1994, 3(4):344-350.

33. Bromham $L$, Rambaut A, Harvey $P$ : Determinants of rate variation in mammalian DNA sequence evolution. Journal of Molecular Evolution 1996, 43(6):610-62I.

34. Martin AP, Naylor GJ, Palumbi SR: Rates of mitochondrial dna evolution in sharks are slow compared with mammals. Nature 1992, 357(6374): I53-I55.

35. Martin AP, Palumbi SR: Body size, metabolic-rate, generation time, and the molecular clock. Proceedings of the National Academy of Sciences of the United States of America 1993, 90(9):4087-4091.

36. Shields GF, Wilson AC: Calibration of mitochondrial DNA evolution in geese. Journal of Molecular Evolution 1987, 24(3):212-217.

37. Bermingham E, Rohwer S, Freeman S, Wood C: Vicariance biogeography in the Pleistocene and speciation in North American wood warblers: a test of Mengel's model. Proceedings of the National Academy of Sciences of the United States of America 1992, 89( I4):6624-6628.

38. Weir JT, Schluter $D$ : The latitudinal gradient in recent speciation and extinction rates of birds and mammals. Science 2007, 3 | 5(58 | 8): | 574-| 576.

39. Lovette If: Mitochondrial dating and mixed-support for the "2\% rule" in birds. Auk 2004, I 2 I: I-6.

40. Garcia-Moreno J: Is there a universal mtDNA clock for birds? Journal of Avian Biology 2004, 35(6):465-468.

4I. Päckert M, Martens J, Tietze DT, Dietzen C, Wink M, Kvist L: Calibration of a molecular clock in tits (Paridae)-do nucleotide substitution rates of mitochondrial genes deviate from the 2\% rule? Molecular Phylogenetics and Evolution 2007, 44:I-I4.

42. Weir JT, Schluter D: Calibrating the avian molecular clock. Molecular Ecology 2008, I 7( I 0):232I-2328.

43. Pereira SL, Baker AJ: A mitogenomic timescale for birds detects variable phylogenetic rates of molecular evolution and refutes the standard molecular clock. Molecular Biology and Evolution 2006, 23(9): |73|- |740.

44. Thorne J, Kishino H, Painter I: Estimating the rate of evolution of the rate of molecular evolution. Molecular Biology and Evolution | 998, I 5(I 2): | 647- |657.

45. Felsenstein J: Phylogenies and the comparative method. American Naturalist 1985, I 25: I- I5.

46. Bazin E, Duret L, Penel S, Galtier N: Polymorphix: a sequence polymorphism database. Nucleic Acids Research 2005, 33:D48I-D484

47. Nevo E, Beiles A, Ben-Shlomo R: The evolutionary significance of genetic diversity: Ecological, demographic and life-history correlates. Lecture Notes in Biomathematics 53: Evolutionary Dynamics of Genetic Diversity 1984.

48. Thorne J, Kishino $\mathrm{H}$ : Divergence time and evolutionary rate estimation with multilocus data. Systematic Biology 2002 5 I(5):689-702.

49. Drummond A, Ho S, Phillips M, Rambaut A: Relaxed phylogenetics and dating with confidence. Plos Biol 2006, 4(5):e88-e88.

50. Yang Z, Rannala B: Bayesian estimation of species divergence times under a molecular clock using multiple fossil calibrations with soft bounds. Molecular Biology and Evolution 2006 , 23:212-226.

51. Thomas J, Welch J, Woolfit M, Bromham L: There is no universal molecular clock for invertebrates, but rate variation does not scale with body size. Proceedings of the National Academy of Sciences of the United States of America 2006, I 03( I 9):7366-737I.

52. Gissi C, Reyes A, Pesole G, Saccone C: Lineage-specific evolutionary rate in mammalian mtDNA. Molecular Biology and Evolution 2000, I7(7): 1022-1031.

53. Castresana J: Cytochrome $b$ phylogeny and the taxonomy of great apes and mammals. Molecular Biology and Evolution 2001, | 8(4):465-47|.
54. Gillooly J, Allen A, West G, Brown J: The rate of dna evolution: effects of body size and temperature on the molecular clock. Proceedings of the National Academy of Sciences of the United States of America 2005, I02: |40-| 45.

55. Ogburn CE, Austad SN, Holmes DJ, Kiklevich JV, Gollahon K, Rabinovitch PS, Martin GM: Cultured renal epithelial cells from birds and mice: enhanced resistance of avian cells to oxidative stress and DNA damage. J Gerontol A Biol Sci Med Sci 1998, 53(4):B287-B292.

56. Herrero $A$, Barja G: $\mathbf{H} 2 \mathrm{o} 2$ production of heart mitochondria and aging rate are slower in canaries and parakeets than in mice: sites of free radical generation and mechanisms involved. Mechanisms of Ageing and Development 1998, I03(2): I33-146.

57. Barja G: Free radicals and aging. Trends in Neurosciences 2004 27( I 0):595-600.

58. Frankham R: Do island populations have less genetic variation than mainland populations? Heredity 1997, 78:3। I-327.

59. Spielman D, Brook B, Frankham R: Most species are not driven to extinction before genetic factors impact them. Proceedings of the National Academy of Sciences of the United States of America 2004, I 0 I (42): I526I-I5264

60. Popadin K, Polishchuk LV, Mamirova L, Knorre D, Gunbin K: Accumulation of slightly deleterious mutations in mitochondrial protein-coding genes of large versus small mammals. Proceedings of the National Academy of Sciences of the United States of America 2007, I 04(33): I3390-13395.

61. Rand D, Kann L: Excess amino acid polymorphism in mitochondrial dna: contrasts among genes from drosophila, mice, and humans. Molecular Biology and Evolution 1996, I3(6):735-748

62. Stanley SE, Harrison RG: Cytochrome b evolution in birds and mammals: an evaluation of the avian constraint hypothesis. Molecular Biology and Evolution 1999, I6(I I): I575-I585.

63. Gillespie J: Is the population size of a species relevant to its evolution? Evolution 200I, 55(I I):216I-2I69.

64. Nei M, Graur D: Extent of protein polymorphism and the neutral mutation theory. Evolutionary Biology 1984, I7:73-1 I8.

65. lizuka $M$, Tachida $H$, Matsuda $H$ : A neutral model with fluctuating population size and its effective size. Genetics 2002 | 61 1:381-388

66. Thompson JD, Higgins DG, Gibson TJ: CLUSTAL W: improving the sensitivity of progressive multiple sequence alignment through sequence weighting, position-specific gap penalties and weight matrix choice. Nucleic Acids Research 1994 22(22):4673-4680.

67. Guindon S, Gascuel O: A simple, fast, and accurate algorithm to estimate large phylogenies by maximum likelihood. Systematic Biology 2003, 52(5):696-704.

68. Ericson PGP, Anderson CL, Britton T, Elzanowski A, Johansson US, Kallersjo M, Ohlson JI, Parsons TJ, Zuccon D, Mayr G: Diversification of neoaves: integration of molecular sequence data and fossils. Biology Letters 2006, 2(4):543-547.

69. Slack KE, Delsuc F, Mclenachan PA, Arnason U, Penny D: Resolving the root of the avian mitogenomic tree by breaking up long branches. Molecular Phylogenetics and Evolution 2007, 42:1-13.

70. Rannala B, Yang Z: Inferring speciation times under an episodic molecular clock. Systematic Biology 2007, 56(3):453-466.

7I. Yang Z: Computional Molecular Evolution Oxford University Press; 2007.

72. Fain $M G$, Houde $P$ : Parallel radiations in the primary clades of birds. Evolution 2004, 58(I I ):2558-2573.

73. Gibb GC, Kardailsky O, Kimball RT, Braun EL, Penny D: Mitochondrial genomes and avian phylogeny: complex characters and resolvability without explosive radiations. Molecular Biology and Evolution 2007, 24:269-280

74. Morgan-Richards M, Trewick SA, Bartosch-Härlid A, Kardailsky $O$ Phillips MJ, Mclenachan PA, Penny D: Bird evolution: testing the Metaves clade with six new mitochondrial genomes. $B M C$ Evolutionary Biology 2008, 8:20-20.

75. Barker FK, Cibois A, Schikler P, Feinstein J, Cracraft J: Phylogeny and diversification of the largest avian radiation. Proceedings of the National Academy of Sciences of the United States of America 2004 I 0 I (30): I I 040-I I 045

76. Dutheil ], Gaillard S, Bazin E, Glemin S, Ranwez V, Galtier N, Belkhir $\mathrm{K}$ : Bio++: a set of $\mathrm{C++}$ libraries for sequence analysis, phylo- 
genetics, molecular evolution and population genetics. $B M C$ Bioinformatics 2006, 7:188-188.

77. Tajima F: Statistical-method for testing the neutral mutation hypothesis by DNA polymorphism. Genetics 1989, I 23(3):585-595.

78. Watterson G: Number of segregating sites in genetic models without recombination. Theoretical Population Biology 1975, 7(2):256-276.

79. de Magalhaes JP, Costa J, Toussaint O: Hagr: the human ageing genomic resources. Nucleic Acids Research 2005, 33:D537-D543.

80. Sokal R, Rohf F: Biometry: The Principles and Practices of Statistics in Biological Research W. H. Freeman; 1994.

81. Team RDC: R: A language and environment for statistical computing. R Foundation for Statistical Computing; 2004.

Publish with Bio Med Central and every scientist can read your work free of charge

"BioMed Central will be the most significant development for disseminating the results of biomedical research in our lifetime. "

Sir Paul Nurse, Cancer Research UK

Your research papers will be:

- available free of charge to the entire biomedical community

- peer reviewed and published immediately upon acceptance

- cited in PubMed and archived on PubMed Central

- yours - you keep the copyright

Submit your manuscript here:

http://www.biomedcentral.com/info/publishing_adv.asp 